\title{
Evaluation of spinopelvic parameters in lumbar prolapsed intervertebral disc
}

\author{
Apoorva Poonia, Sambhav Lodha, N C Sharma \\ Department of Radiology, Geetanjali Medical College and Hospital, Udaipur, Rajasthan, India \\ Correspondence: Dr. Apoorva Poonia, 146, VPO Dhansa, New Delhi - 110 073, India. E-mail: pooniapoorva@gmail.com
}

\begin{abstract}
Background: Sacro-pelvic morphology and orientation are usually described in terms of pelvic incidence (PI), pelvic tilt (PT), and sacral slope (SS). Orientation and morphology of pelvis can affect degenerative changes in the lumbar spine. Thus, here we investigated the relationships between various sagittal spinopelvic parameters and the degree of disc degeneration in young adults. Material and Methods: A hospital-based cross-sectional study with a total of 60 cases was done. Patients presenting with back or leg pain having prolapsed disc on magnetic resonance imaging (MRI) were included in the study. A standing X-ray of LS spine from dorso-lumbar junction to mid-thigh was taken. Various spinopelvic parameters were assessed from the scannogram using the software. Results: The mean age was 39.27 years. L5S1 was the most common level. Mean SS, PT, PI, and LL were $37.78^{\circ}, 13.52^{\circ}, 51.33^{\circ}$, and $41.01^{\circ}$. Disc pathologies at $\mathrm{L} 1 \mathrm{~L} 2, \mathrm{~L} 2 \mathrm{~L} 3$, and $\mathrm{L} 4 \mathrm{~L} 5$ level showed a positive correlation with $\mathrm{PT}, \mathrm{PI}$, and LL. Disc pathology at the L5S1 level shows a positive correlation with PT and LL. A statistically significant correlation between SS and degenerative spondylolisthesis at L4L5 was found from data with $P=0.023$. Discussion: An increase in SS statistically significantly increases the chance of development of degenerative spondylolisthesis at L4L5. An increase in PT, PI, and LL will cause an increase in disc pathology at L1L2. An increase in SS, PT, PI, and LL will cause an increase in disc pathology at L2L3. An increase in SS, PT, PI, and LL will cause an increase in disc pathology at L4L5. An increase in PT and LL will cause an increase in disc pathology at L5S1. Conclusion: Standing lateral view radiograph from dorso-lumbar junction to the mid-thigh is as good as standing whole spine radiograph for measurement of spinopelvic parameters. Degenerative spondylolisthesis at L4L5 has a statistically significant correlation with an increase in SS.
\end{abstract}

Key words: Lumbar disc herniation; pelvic incidence; pelvic tilt; sacral slope; spinopelvic parameters

\section{Introduction}

The human lumbar spine consists of five lumbar vertebrae articulating with each other at intervertebral discs anteriorly and facets joints posteriorly. Intervertebral disc functions physiologically as a shock absorber of the spine and helps to maintain the lordotic curve of lumbar spine. The functional and clinical importance of lumbar lordosis is being recognized increasingly. ${ }^{[1,2]}$ Loss of normal lordotic alignment may induce pathologic changes in the spine from load-bearing

\begin{tabular}{|l|l|}
\hline \multicolumn{2}{|c|}{ Access this article online } \\
\hline Quick Response Code: & \\
\hline & Website: \\
& www.ijri.org \\
& DOI: \\
&
\end{tabular}

and accelerate degeneration of the functional motion units. ${ }^{[3]}$ The lumbar spine rests on the first sacral vertebra which is an integral part of the pelvis. The lumbar spine and pelvis are biomechanically connected, pathology of one affecting other, and vice-versa. Orientation and morphology of pelvis can, therefore, affect degenerative changes in the lumbar spine due to altered biomechanical forces.

This is an open access journal, and articles are distributed under the terms of the Creative Commons Attribution-NonCommercial-ShareAlike 4.0 License, which allows others to remix, tweak, and build upon the work non-commercially, as long as appropriate credit is given and the new creations are licensed under the identical terms.

For reprints contact: WKHLRPMedknow_reprints@wolterskluwer.com

Cite this article as: Poonia A, Lodha S, Sharma NC. Evaluation of spinopelvic parameters in lumbar prolapsed intervertebral disc. Indian J Radiol Imaging 2020;30:253-62.

Received: 14-Feb-2020 Revised: 10-Apr-2020

Accepted: 14-Jun-2020 Published: 15-Oct-2020 
Sacropelvic morphology refers to the anatomy (shape) specific to each individual. On the contrary, sacropelvic orientation depends on the position of the individual in space and is best measured from standing lateral radiographs with the hips and knees extended [Figure 1]. Sacro-pelvic morphology and orientation are usually described in terms of pelvic incidence (PI), pelvic tilt (PT), and sacral slope (SS). The PI is a morphological parameter describing the sacro-pelvis, which is specific and constant for each individual. This parameter introduced by Duval-Beaupère et al..$^{[4]}$ is defined as the angle between the line perpendicular to the upper sacral endplate and the line joining the middle of the upper sacral endplate and the hip axis [Figure 2]. The mean value of PI in the Indian population as given by Singh et al.$^{[5]}$ is $48.52 \pm 8.99$. In contrast to the PI, the PT and SS measure the orientation of the Sacro-pelvis in the sagittal plane. SS is defined as the angle between the sacral

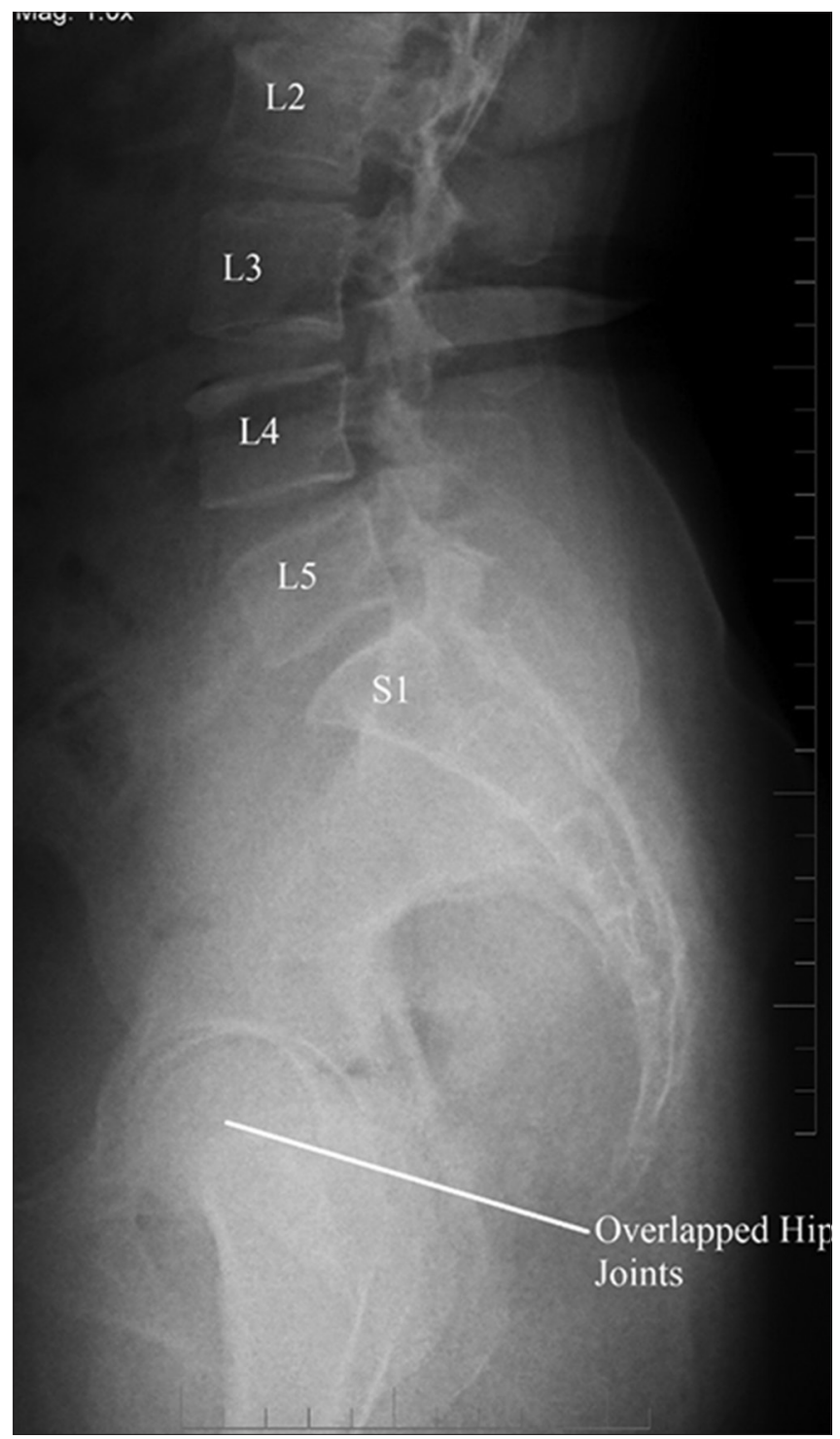

Figure 1: Lateral view lumbo-sacral spine with hip joints overlapped and extended. (patient in standing position) endplate and the horizontal reference line (HRL) [Figure 2], whereas PT is defined as the angle between the vertical reference line (VRL) and the line joining the middle of the sacral endplate and the hip axis [Figure 2]. The mean values of SS and PT in the Indian population are $39.14 \pm 7.05$ and $9.30 \pm 7.16$, respectively. ${ }^{[5]}$ PI represents the arithmetic sum of the PT and SS (PI = PT + SS).

In a static standing position, the way SS and PT balance themselves refers to the concept of acro-pelvic balance. Patients with high PI and SS would increase the shear stresses at the lumbosacral junction, causing more tension on L5S1 articulations, i.e., intervertebral disc anteriorly and facets joints posteriorly. This increased stress, in theory, will increase disc degeneration and prolapse at this level [Figure 3].

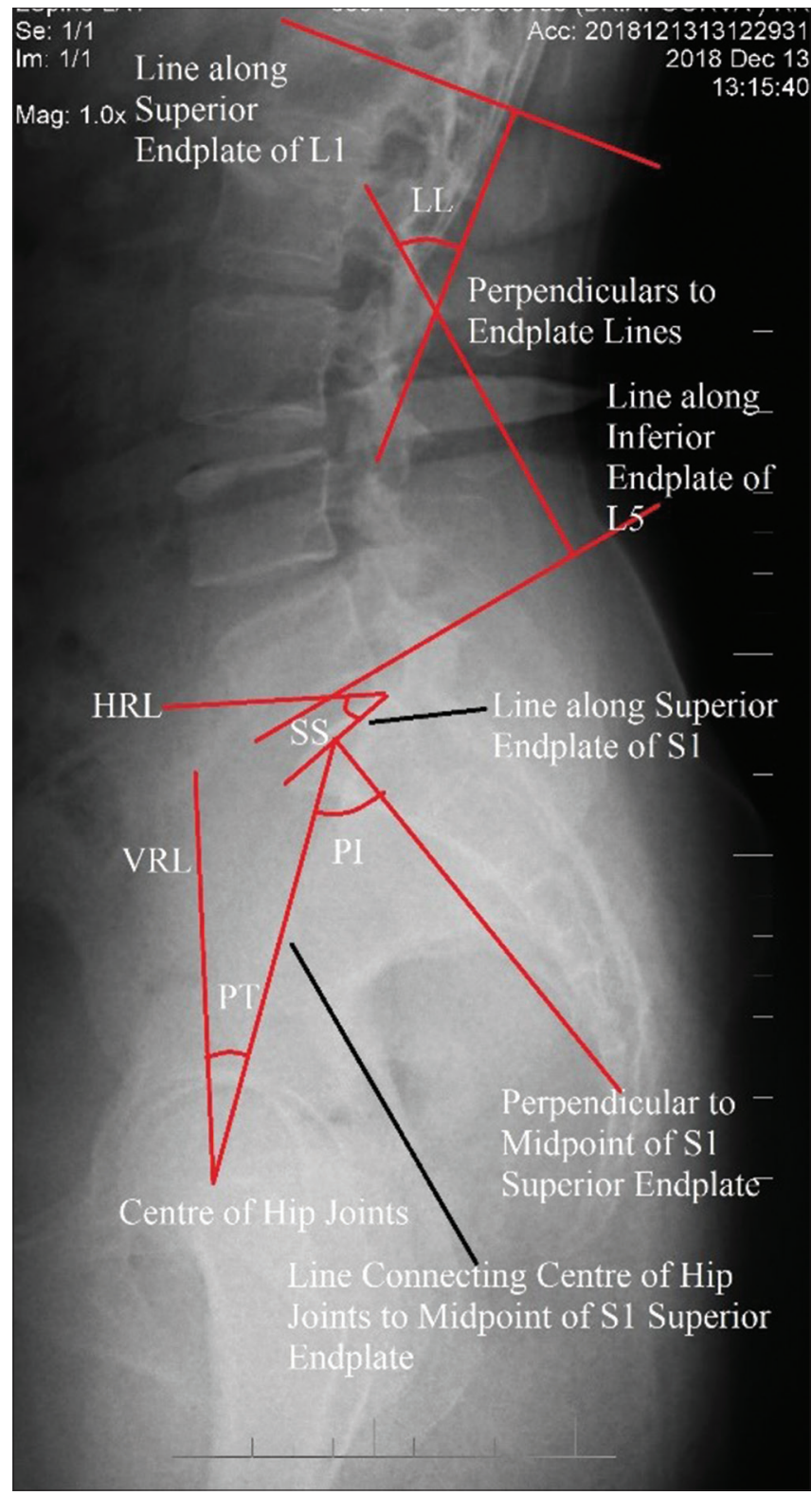

Figure 2: Measurement of PI, SS, PT, and LL 
In normal individuals, studies have shown that the sacropelvic morphology determines the sacro-pelvic orientation, which in turn greatly influences the shape and orientation of the spine, especially the lumbar lordosis. ${ }^{[6,7]}$ This results in an open linear chain linking the head to the pelvis where the shape and orientation of each successive anatomical segment are closely related and influence the adjacent segment $t^{[-8]}$ to maintain the center of gravity over the femoral heads. Hence, any change in SS will affect LL. The normal physiological orientation of the lumbar spine is that of lordosis which ranges between $40^{\circ}$ and $60^{\circ}$. Change in lumbar lordosis outside of normal range affects load transmission along the lumbar spine and hence leads to accelerated disc degeneration. Recently, Keorochana et al. ${ }^{[3]}$ reported that sagittal spinopelvic alignment may alter spinal load and mobility, thereby possibly influencing segmental degeneration; moreover, alterations in sagittal spinopelvic alignment may lead to kinematic changes that influence load bearing and the distribution of disc degeneration at each level. Consequently, the analysis of sagittal balance recently seems to be essential in the

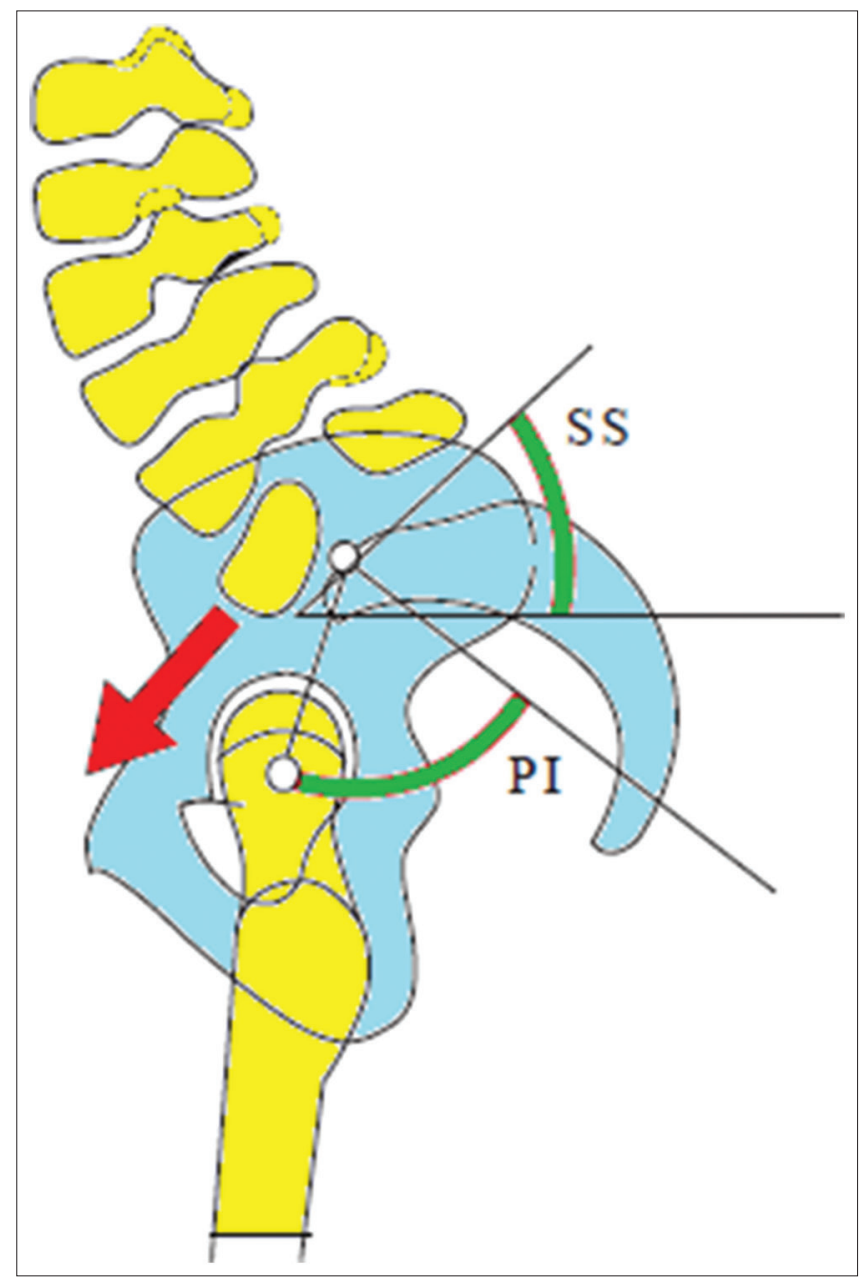

Figure 3: Increase in $\mathrm{PI}$ and henceforth SS will cause increased shear stress on lumbo-sacral junction and will accelerate degenerative changes management of lumbar degenerative pathologies. ${ }^{[9]}$ Nevertheless, the relationship between sagittal balance and the degree of disc degeneration has not been extensively explored. Thus, here we investigated the relationships between various sagittal spinopelvic parameters and the degree of disc degeneration in young adults. LL is measured as an angle between lines along the superior endplate of the L1 vertebra and the inferior endplate of the L5 vertebra [Figure 2]. The mean value of LL in the Indian population is $58.78 \pm 9.51 .^{[5]}$

\section{Material and Methods}

Our study was a hospital-based cross-sectional study done in the Department of Radiodiagnosis, GMCH, Udaipur from November 2017 to April 2020. A total of 60 cases were included in the study. All outdoor patients of age between 18 and 50 years with chronic prolapsed intervertebral disc visiting the Department of Radiodiagnosis, GMCH, Udaipur were recruited to participate in the study. All patients were evaluated clinically and radiologically with X-ray and MRI. Inclusion criteria were individual of $18-50$ years age group having back or leg pain without any history of other spinal disease or deformity, having prolapsed intervertebral disc on MRI. Criteria for exclusion were - Patients not consenting for the study, patients with a history of trauma, pregnant females, patients with scoliotic deviation, and patients with congenital anomalies, post-polio residual paralysis, and neuromuscular dystrophy. Institutional Ethical Committee approval and prior informed consent were taken from all patients.

Patients presenting with back or leg pain having prolapsed disc on MRI were included in the study. A standing X-ray of LS spine from dorso-lumbar junction to mid-thigh was taken. Each patient was asked to stand in his or her own neutral standing position. The knees were kept straight. The arms were placed over the chest in such a way as to remove arms from the field of view [Figure 4]. Various spinopelvic parameters were assessed from the scannogram using software (Digimizer Image Analysis Software, Version 5.4.4; (C) 2005-2020 MedCalc Software Ltd).

\section{Results}

Our study population comprised young adults in the age group between 20 years to 50 years. The mean age was 39.27 years with a standard deviation of \pm 8.93 . The maximum number of patients was in the age group between 41 and 50 years. Females comprised of major sex group among the study population. There were 37 female patients making $61.7 \%$ of the total group as compared to 23 male patients making $38.3 \%$ of the total population. Patients with two levels of disc pathologies were major group comprising of $58.3 \%$ of cases. L5S1 was the most common level followed by L4 L5 in cases with a single level of disc pathologies. 
L4L5 + L5S1 were the most commonly involved levels in cases with two-level involvement however L4L5 was the most common level involved in combination with other levels in cases with two-level involvement. Diffuse disc bulge was the commonest type of disc pathology comprising $31(51.7 \%)$ cases. Disc protrusion was the second commonest type with $20(33.3 \%)$ cases.

\section{SS, PT, PI, and LL}

Mean SS in the study population was $37.78^{\circ}$ with a standard deviation of \pm 9.34 [Table 1]. The median value of SS was $37.08^{\circ}$ with a range of 13.11 to 58.39 . Mean PT in the study population was $13.52^{\circ}$ with a standard deviation of \pm 5.84 . The median value of PT was $12.64^{\circ}$ with a range of 1.52 to 25.24 . Mean PI in the study population was $51.33^{\circ}$ with a standard deviation of \pm 9.19 . The median value of PI was $50.94^{\circ}$ with a range of 30.44 to 71.62 . Mean LL in the study population was $41.01^{\circ}$ with a standard deviation of \pm 14.52 . The median value of SS was $42.46^{\circ}$ with a range of $1.51-70.28$. The sum of the mean of SS and PT $(37.78+13.52=51.3)$ is equal to PI mean (51.33). So, the correlation between SS, PT, and PI that is $\mathrm{PI}=\mathrm{PT}+\mathrm{SS}$ stands true in the result of our study.

\section{Correlation between SS, PT, and PI}

Pearson correlation shows that SS, PT, and PI are linearly correlated. A change in the value of one will correspondingly

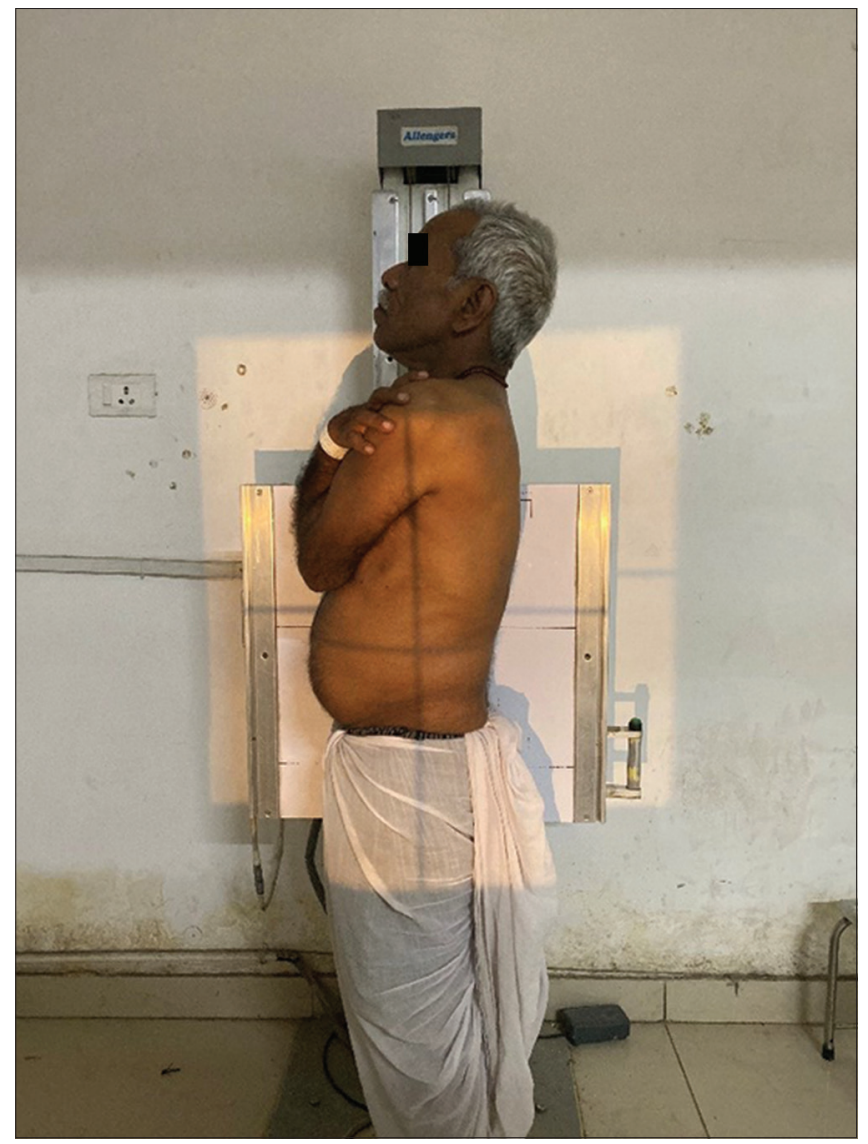

Figure 4: Position of patient for taking radiograph change the other two linearly [Table 2 and Figures 5-7]. This linear correlation is statistically significant in all the cases with $P<0.05$. SS had positive linear correlation with PI (Pearson correlation coefficient $=0.798$ ) and negative linear correlation with PT (Pearson correlation coefficient $=-0.303)$. Similarly, PT had a positive linear correlation with PI (Pearson correlation coefficient $=0.330$ ) and a negative linear correlation with SS (Pearson correlation coefficient $=-0.303)$. PI had positive linear correlation with both

Table 1: Values of SS, PT, PI, and LL in study population

\begin{tabular}{lccc}
\hline & Mlean \pm SD & Median & IVin-IMax \\
\hline SS & $37.78 \pm 9.34$ & 37.08 & $13.11-58.39$ \\
PT & $13.52 \pm 5.84$ & 12.64 & $1.52-25.24$ \\
PI & $51.33 \pm 9.19$ & 50.94 & $30.44-71.62$ \\
LL & $41.01 \pm 14.52$ & 42.46 & $1.51-70.28$ \\
\hline
\end{tabular}

Table 2: Pearson correlation (linear correlation) between SS, PT, and PI

\begin{tabular}{lccc}
\hline \multicolumn{3}{c}{ Correlations } & \\
\hline SS & SS & PT & \\
Pearson correlation & 1 & $-0.303^{*}$ & $0.798^{* *}$ \\
$P$ & & 0.019 & $<0.001$ \\
$n$ & 60 & 60 & 60 \\
PT & & & \\
Pearson correlation & $-0.303^{*}$ & 1 & $0.330^{* *}$ \\
$P$ & 0.019 & & 0.010 \\
$n$ & 60 & 60 & 60 \\
PI & & & 1 \\
Pearson correlation & $0.798^{* *}$ & $0.330^{* *}$ & \\
$P$ & $<0.001$ & 0.010 & 60 \\
$N$ & 60 & 60 & 60 \\
$N$ & 60 & 60 & \\
\hline
\end{tabular}

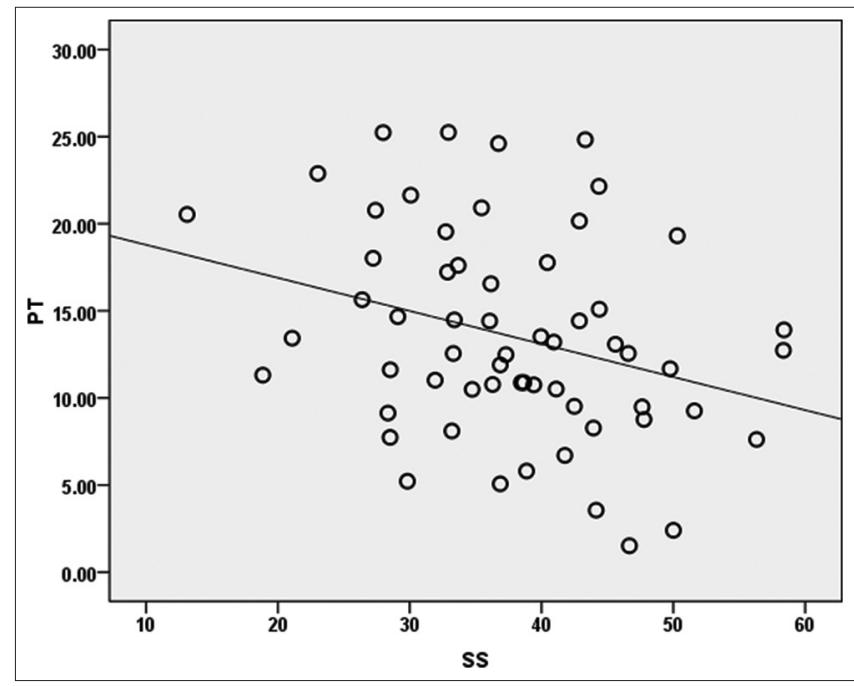

Figure 5: Pearson correlation (linear correlation) between SS and PT 
SS (Pearson correlation coefficient $=0.798)$ and PT $($ Pearson correlation coefficient $=0.330$ ).

Correlation between LL and SS, PT, and PI

LL was found to be linearly correlated to SS and in turn to PI and this correlation was statistically significant with $P<0.05$. No such statistically significant correlation was found between LL and PT [Table 3 and Figures 8-10].

\section{Correlation between Disc pathology at L1L2 and pelvic} parameters (SS, PT, PI, and LL)

In cases with L1L2 disc pathology, mean SS, PT, PI, and LL were $36.59 \pm 8.23,16.22 \pm 5.06,53.18 \pm 7.10$ and $41.07 \pm 13.90$, respectively [Figure 11]. Disc pathologies at L1L2 level showed positive monotonic correlation with PT, PI, and LL (Spearman's rho correlation coefficient of 0.173, 0.083, and 0.016 , respectively). These correlations however were not statistically significant [Table 4].

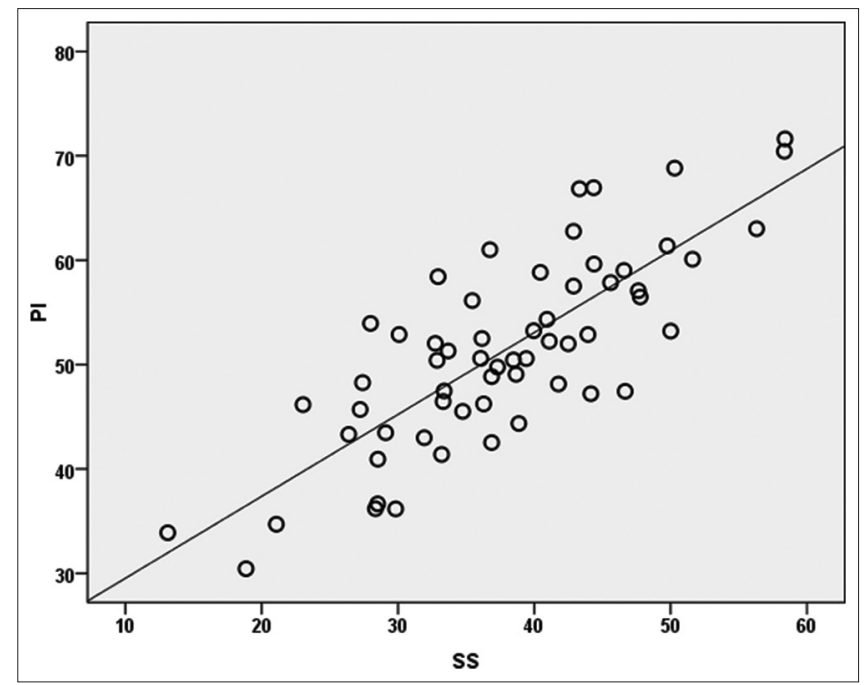

Figure 6: Pearson correlation (linear correlation) between SS and $\mathrm{PI}$

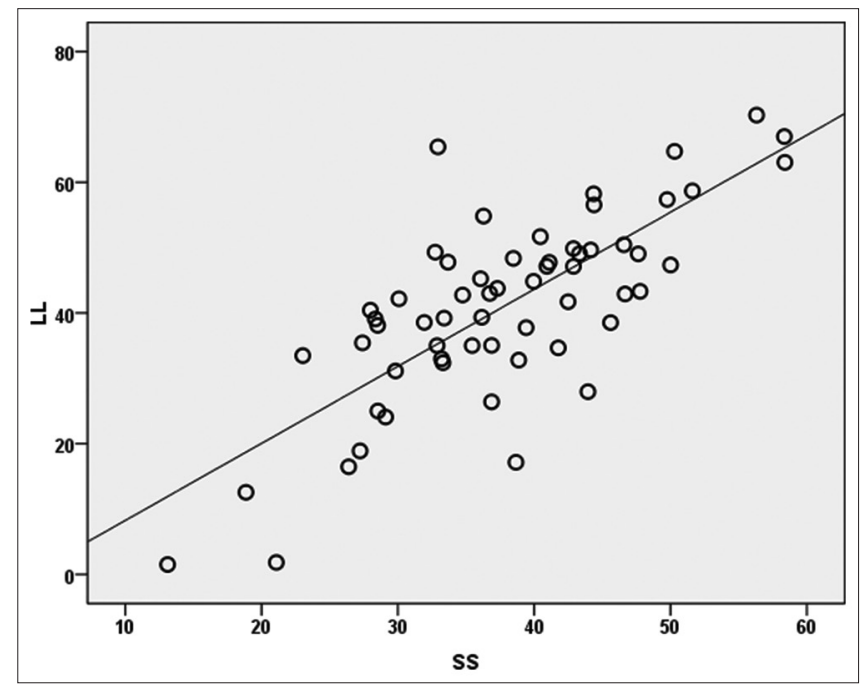

Figure 8: Pearson correlation (linear correlation) between LL and SS
Correlation between Disc pathology at L2L3 and pelvic parameters (SS, PT, PI, and LL)

In cases with L2L3 disc pathology, mean SS, PT, PI, and LL were $39.42 \pm 9.26,13.86 \pm 8.09,53.36 \pm 8.39$ and $46.92 \pm 13.89$, respectively [Figure 12]. Disc pathologies at the L2L3 level showed positive monotonic correlation with SS, PT, PI, and LL (Spearman's rho correlation coefficient of 0.031, 0.042, 0.074 , and 0.136 , respectively). These correlations however were not statistically significant [Table 4].

Table 3: Pearson correlation (linear correlation) between LL and SS, PT, PI

\begin{tabular}{lccc}
\hline \multicolumn{4}{c}{ Correlations } \\
\hline LL & SS & PT & PI \\
Pearson correlation & $0.759^{* *}$ & 0.049 & $0.781^{* *}$ \\
$P$ & $<0.001$ & 0.712 & $<0.001$ \\
$n$ & 60 & 60 & 60 \\
\hline
\end{tabular}

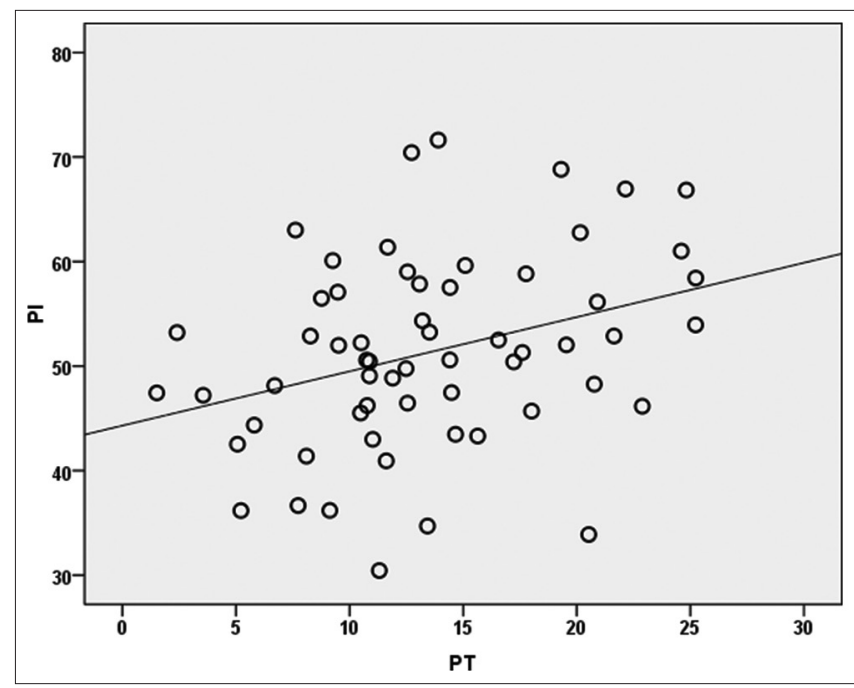

Figure 7: Pearson correlation (linear correlation) between PT and PI

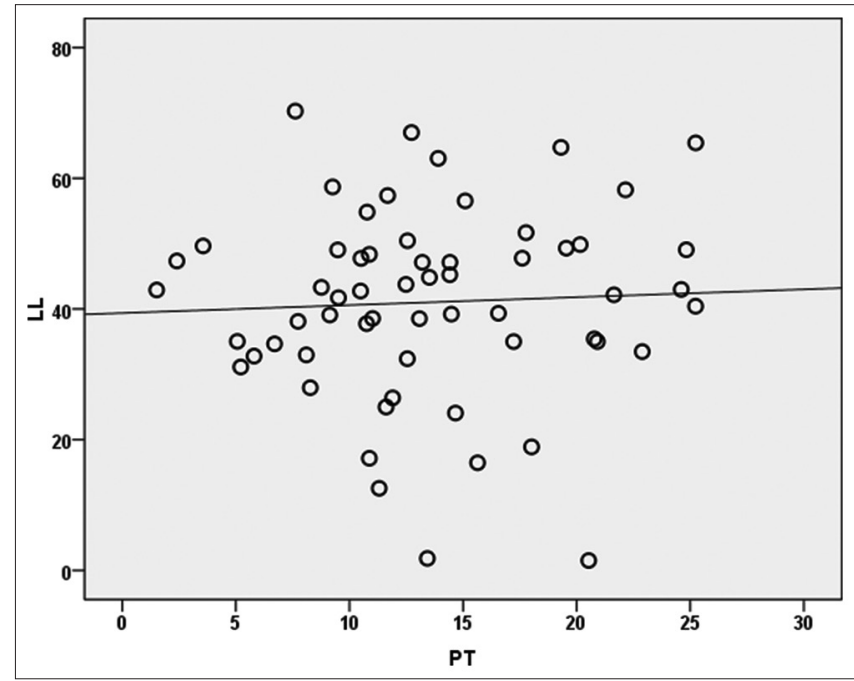

Figure 9: Pearson correlation (linear correlation) between LL and PT 
Correlation between disc pathology at L3L4 and pelvic parameters (SS, PT, PI, and LL)

In cases with L3L4 disc pathology, mean SS, PT, PI, and LL were $37.41 \pm 6.21,13.86 \pm 7.56,51.34 \pm 5.39$ and $42.09 \pm 7.49$, respectively [Figure 13]. Disc pathology at L3L4 level show positive monotonic correlation with LL (Spearman's rho correlation coefficient of 0.027 ) only and shows a negative correlation with SS, PT, and PI (Spearman's rho correlation coefficient of $-0.035,-0.013$, and -0.004 , respectively). These correlations however were not statistically significant [Table 4].

Correlation between Disc pathology at L4L5 and pelvic parameters (SS, PT, PI, and LL)

In cases with L4L5 disc pathology, mean SS, PT, PI, and LL were $38.36 \pm 9.43,13.58 \pm 5.77,51.93 \pm 9.69$, and $41.92 \pm 15.06$, respectively [Figure 14]. Disc pathology at L4L5 level shows a positive monotonic correlation with SS, PT, PI, and LL (Spearman's rho correlation coefficient of $0.106,0.014,0.086$, and 0.106 , respectively). These correlations however were not statistically significant [Table 4].

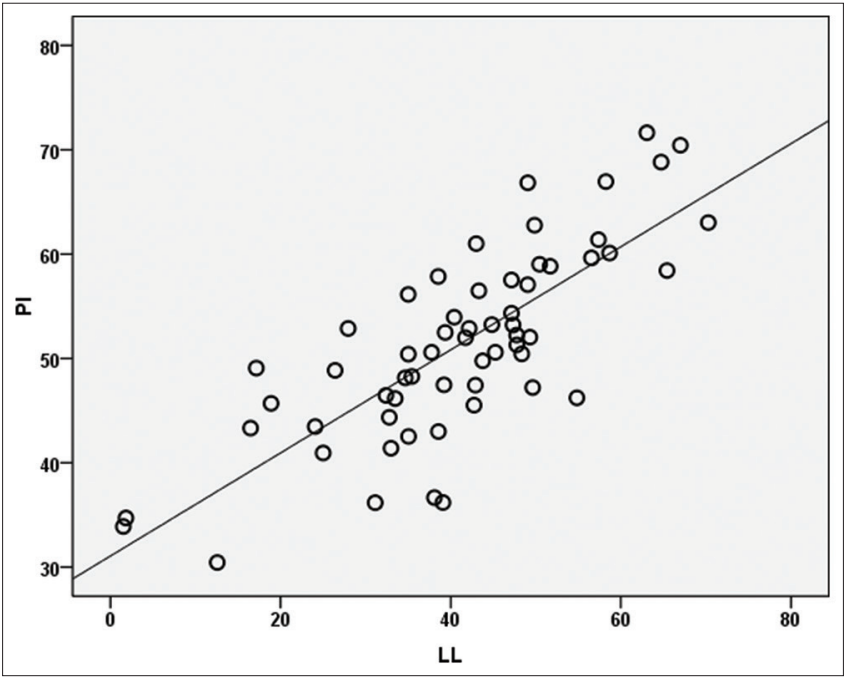

Figure 10: Pearson correlation (linear correlation) between LL and PI

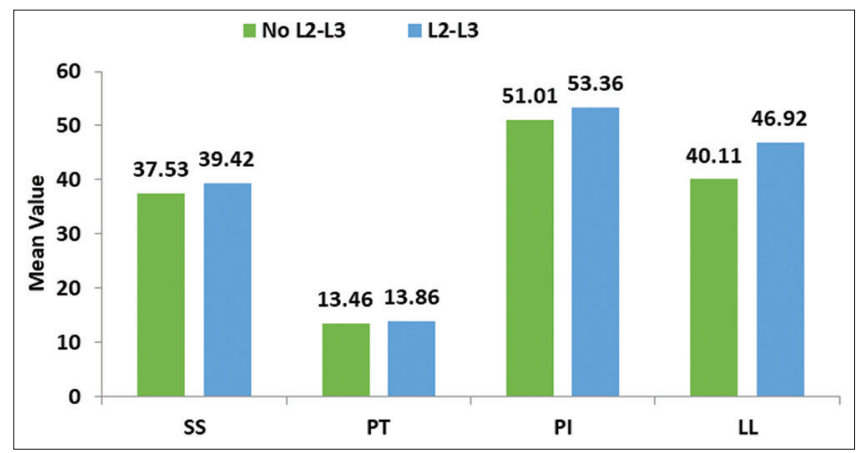

Figure 12: Comparison of mean and median values of SS, PT, PI, and LL among cases with disc pathology at L2L3 and no disc pathology at L2L3
Correlation between Disc pathology at L5S1 and pelvic parameters (SS, PT, PI, and LL)

In cases with L5S1 disc pathology, mean SS, PT, PI, and LL were $37.44 \pm 10.35,13.63 \pm 5.10,51.06 \pm 10.23$, and $40.49 \pm 15.18$, respectively [Figure 15]. Disc pathology at L5S1 level shows a positive monotonic correlation with PT and LL (Spearman's rho correlation coefficient of 0.038 and 0.003, respectively). Disc pathology at L5S1 however showed a negative monotonic correlation with PI (Spearman's rho correlation coefficient of -0.004 ). These correlations however were not statistically significant [Table 4].

Correlation between degenerative spondylolisthesis at L4L5 and SS, PT, PI, LL

A total of four (6.7\%) cases had degenerative listhesis and all of them had it at the level of L4L5. A statistically significant correlation between SS and degenerative spondylolisthesis at L4L5 was found from data with $P=0.023$ [Table 5]. This correlation was found to be a monotonic direct correlation with Spearman's rho correlation coefficient of 0.293 [Table 6]. A similar trend is shown by PI (Spearman's rho correlation coefficient of 0.177 ) and LL (Spearman's rho correlation coefficient of 0.201) i.e., increase in PI and LL lordosis increase chance of development of degenerative spondylolisthesis at L4L5 but this increase was not found to be statistically significant for PI and LL.

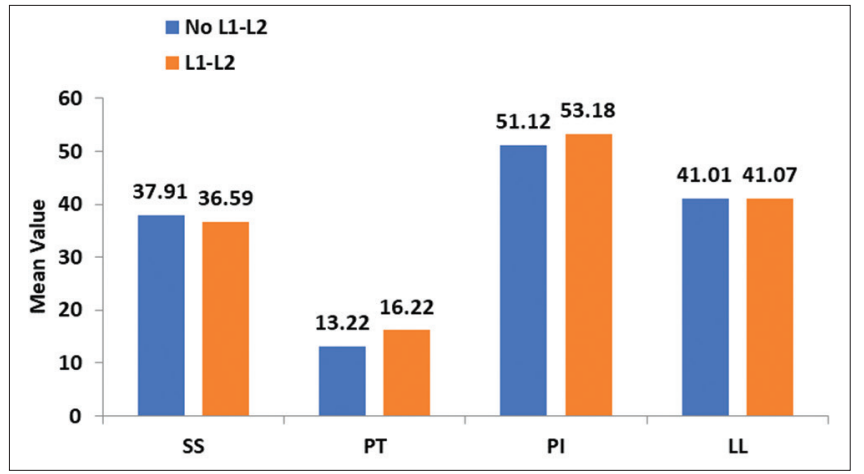

Figure 11: Comparison of mean and median values of SS, PT, PI, and LL among cases with disc pathology at L1L2 and no disc pathology at L1L2

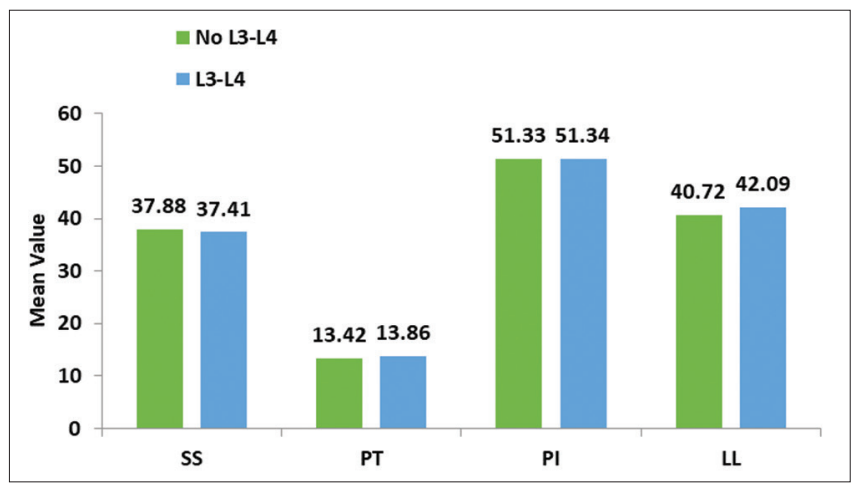

Figure 13: Comparison of mean and median values of SS, PT, PI, and $\mathrm{LL}$ among cases with disc pathology at L3L4 and no disc pathology at L3L4 


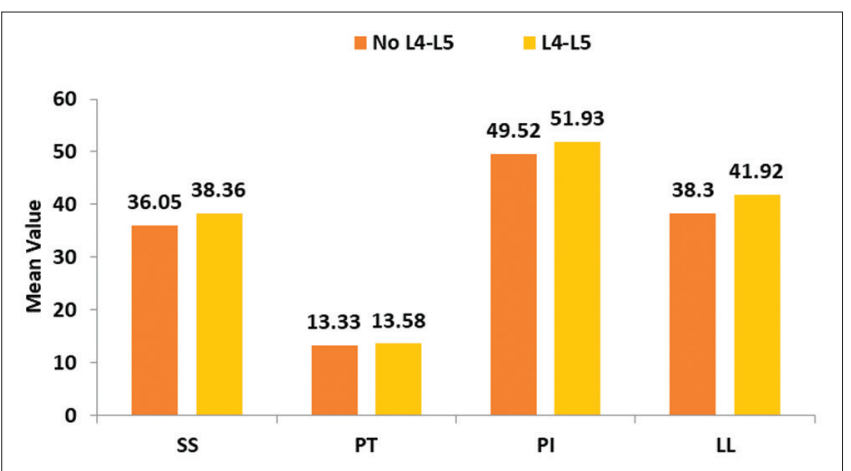

Figure 14: Comparison of mean and median values of SS, PT, PI, and LL among cases with disc pathology at L4L5 and no disc pathology at L4L5

Table 4: Spearman's rank correlation coefficient (Spearman's rho) between disc pathologies at various levels and SS, PT, PI, and LL.

\begin{tabular}{|c|c|c|c|c|c|c|}
\hline \multicolumn{7}{|c|}{ Correlations } \\
\hline \multicolumn{3}{|c|}{ Disc level } & \multirow{2}{*}{$\begin{array}{c}\text { SS } \\
-0.042\end{array}$} & \multirow{2}{*}{$\begin{array}{c}\text { PT } \\
0.173\end{array}$} & \multirow{2}{*}{$\begin{array}{c}\text { PI } \\
0.083\end{array}$} & \multirow{2}{*}{$\begin{array}{c}\text { LL } \\
0.016\end{array}$} \\
\hline $\begin{array}{l}\text { Spearman's } \\
\text { rho }\end{array}$ & L1-L2 & $\begin{array}{l}\text { Correlation } \\
\text { coefficient }\end{array}$ & & & & \\
\hline & & $P$ & 0.752 & 0.186 & 0.526 & 0.903 \\
\hline & & $N$ & 60 & 60 & 60 & 60 \\
\hline & L2-L3 & $\begin{array}{l}\text { Correlation } \\
\text { coefficient }\end{array}$ & 0.031 & 0.042 & 0.074 & 0.136 \\
\hline & & $P$ & 0.813 & 0.747 & 0.576 & 0.301 \\
\hline & & $N$ & 60 & 60 & 60 & 60 \\
\hline & L3-L4 & $\begin{array}{l}\text { Correlation } \\
\text { coefficient }\end{array}$ & -0.035 & -0.013 & -0.004 & 0.027 \\
\hline & & $P$ & 0.79 & 0.922 & 0.979 & 0.839 \\
\hline & & $N$ & 60 & 60 & 60 & 60 \\
\hline & L4-L5 & $\begin{array}{l}\text { Correlation } \\
\text { coefficient }\end{array}$ & 0.106 & 0.014 & 0.086 & 0.106 \\
\hline & & $P$ & 0.422 & 0.913 & 0.516 & 0.422 \\
\hline & & $N$ & 60 & 60 & 60 & 60 \\
\hline & L5-SI & $\begin{array}{l}\text { Correlation } \\
\text { coefficient }\end{array}$ & 0 & 0.038 & -0.04 & 0.003 \\
\hline & & $P$ & 0.994 & 0.775 & 0.764 & 0.982 \\
\hline & & $n$ & 60 & 60 & 60 & 60 \\
\hline
\end{tabular}

\section{Discussion}

The adoption of vertical posture represents the main transformation in the evolution of humans. Spine and spinopelvic complex played a major role in vertical posture development and bipedalism. To achieve this marvellous feat, the human spine comprises of successive, opposing curves that allow the trunk to assume an erect position. The lumbar lordosis is unique and is not found in any other species. Pelvis also underwent tremendous modifications to alter posture to a vertical one. The pelvis attempts to couple lumbar lordosis with hip extension in the erect position with minimal expense of energy. However, some pelvis can accomplish this task better than others. Progressive knowledge has demonstrated that the shape of the pelvis

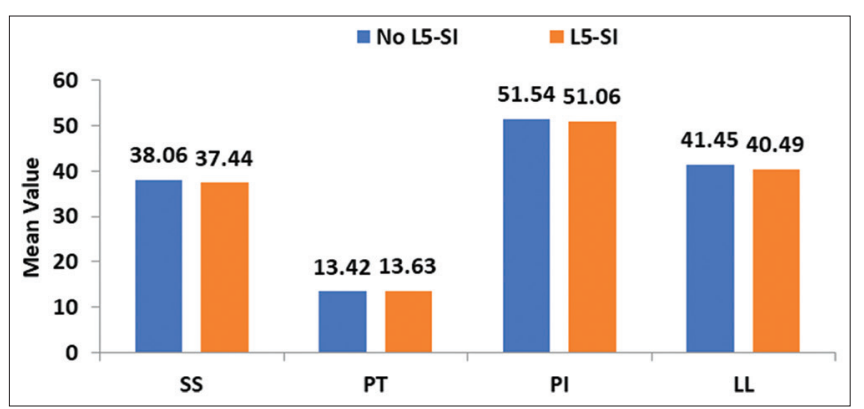

Figure 15: Comparison of mean and median values of SS, PT, PI, and LL among cases with disc pathology at L5S1 and no disc pathology at L5S1

and its relation to the SS influence dramatically the type of lumbar lordosis in a single individual. ${ }^{[10]}$ Due to the work of Duval-Beaupère et al. ${ }^{[4,11,12]}$ it became possible to define the pelvic geometry and the relation of this geometry with the position of the pelvis. Recently, some authors have highlighted the correlation between lumbar spine disc pathologies and degeneration with the spinopelvic organization..$^{[13]}$

The key for it is the pelvic incidence (PI) angle. It is now crystal clear that the PI and the SS are important in determining the type of lumbar lordosis in a specific individual. The specific spinopelvic geometry will result in mechanical stress at the lumbar spine.

Any pattern of the degenerative spine is not a static entity. This is a result of dynamic forces acting on it because of its spatial orientation in space and resulting in biomechanical forces. There is a strong correlation in shape and positioning, and form and function, between the pelvis and the spine. The morphology of the pelvis is identified by PI, with consequences on the morphology of the spine. With time, depending on the individual morphology of a person, specific degenerative evolutions may occur. Sagittal parameters may be considered as predictive regarding the respective shape of the spine and pelvis. A better understanding of this relation may lead to improved diagnosis of degenerative spine diseases and a better strategy of treatment.

The mean age in our study falls in the young adult category. Age group was similar in the study by Endo et al. ${ }^{[14]}$ with a mean age of 32.7 years. Although other studies by P. Rajnics et al..$^{[15]}$ and Barrey et al. ${ }^{[9]}$ constituted a higher age group with a mean age of $47.70 \pm 14.15$ years and $49 \pm 12$ years, respectively. Sex distribution in our study was female dominant as was in the study by Barrey et al..$^{[9]}$ while in a study by Endo et al..$^{[14]}$ males formed a majority group.

A statistically significant monotonic direct correlation of SS with degenerative spondylolisthesis at L4L5 was found in our study. A monotonic direct relationship is where an 
Table 5: Correlation between degenerative spondylolisthesis at L4L5 and SS, PT, PI, LL.

\begin{tabular}{|c|c|c|c|c|c|}
\hline & \multicolumn{2}{|c|}{ Degenerative spondylolisthesis L4L5 } & \multicolumn{2}{|c|}{ Spondylolisthesis $=$ No } & \multirow[t]{2}{*}{$P$} \\
\hline & Mean \pm SD & Median (IOR) & Mean \pm SD & Median (IQR) & \\
\hline SS & $47.97 \pm 7.13$ & $45.51(42.96-55.43)$ & $37.05 \pm 9.10$ & $36.81(30.54-43.21)$ & 0.023 \\
\hline PT & $11.48 \pm 8.53$ & $11.12(3.52-19.79)$ & $13.67 \pm 5.69$ & $12.82(9.73-17.73)$ & 0.475 \\
\hline $\mathrm{PI}$ & $59.20 \pm 11.20$ & $59.47(48.57-69.56)$ & $50.77 \pm 8.88$ & $50.58(45.56-57.40)$ & 0.076 \\
\hline LL & $52.47 \pm 12.26$ & $50.57(42.02-64.81)$ & $40.20 \pm 14.41$ & $41.29(33.10-49.05)$ & 0.103 \\
\hline
\end{tabular}

Table 6: Spearman's rank correlation coefficient (Spearman's rho) between degenerative spondylolisthesis at L4L5 and SS, PT, PI, LL

\begin{tabular}{lcccc}
\hline \multicolumn{5}{c}{ Correlations } \\
\hline & SS & PT & PI & LL \\
\hline Spearman's rho & & & & \\
Spondylolisthesis & & & & \\
$\quad$ Correlation coefficient & $0.293^{*}$ & -0.073 & 0.177 & 0.201 \\
$P$ & 0.023 & 0.578 & 0.175 & 0.124 \\
$n$ & 60 & 60 & 60 & 60 \\
\hline
\end{tabular}

increase in the independent variable causes an increase in the dependent variable; never to remain constant or decrease, i.e., our study signifies that an increase in SS statistically significantly increases the chance of development of degenerative spondylolisthesis at L4L5. A similar trend is shown by PI and LL although it was not statistically significant. Similar findings were reported by Wang et al. ${ }^{[16]}$ They reported that cases with single-level degenerative spondylolisthesis have higher PI and SS than those without degenerative spondylolisthesis. Ferrero et al. ${ }^{[17]}$ reported that patients with degenerative spondylolisthesis had higher PI as compared to asymptomatic volunteers. Similarly, Lai et al. ${ }^{[18]}$ found that PI was associated with degenerative spondylolisthesis and that among patients with degenerative spondylolisthesis, SS has higher values compared to a control group.

A statistically significant linear correlation was found between SS, PI, and PT [Table 2]. In our study, increase in SS linearly increased PI as PI is the mathematical sum of SS and PT. On the contrary, an increase in SS decreased PT because the pelvis has to compensate for increase SS to maintain a straight posture of the body, and pelvis does this by reducing its tilt, i.e., PT. Increase in PT linearly increased PI as PI is mathematical sum of PT and SS. On the contrary, an increase in PT decreased SS because the spine has to compensate for increase PT to maintain a straight posture of body and spine does this by reducing SS i.e., SS.

LL showed a statistically significant linear correlation with SS and PI. Any increase in SS will increase LL. This is on expected lines to compensate for increased SS, the lumbar spine will have to increase its curve to maintain an erect standing posture.

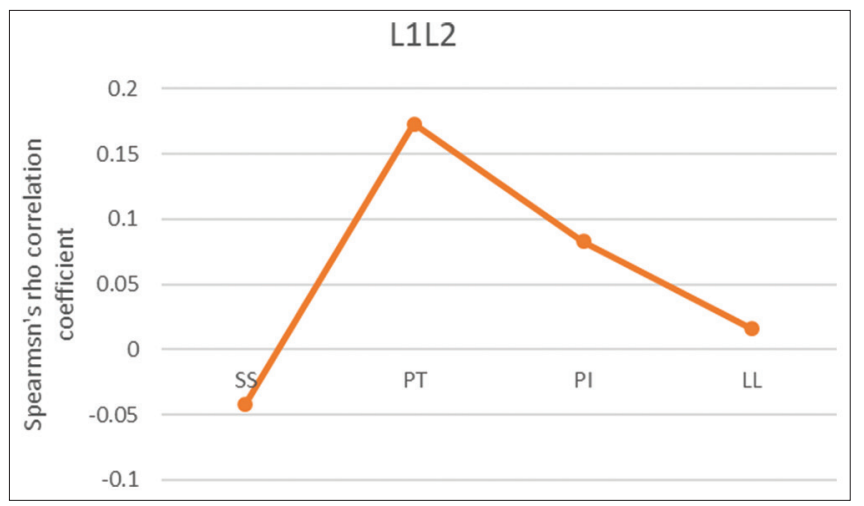

Figure 16: Correlation between disc pathology at L1L2 and spino-pelvic parameters

Disc pathologies at the L1L2 level showed a positive monotonic correlation with PT, PI, and LL [Figure 16]. It implies that an increase in PT, PI, and LL will cause an increase in disc pathology at L1L2 in a monotonic fashion, i.e., disc pathology at L1L2 always increasing; never remaining constant or decreasing with increase in PT, PI, and LL.

Disc pathologies at L2L3 and L4L5 level showed positive monotonic correlation with SS, PT, PI, and LL [Figures 17 and 18]. It implies that an increase in SS, PT, PI, and LL will cause an increase in disc pathology at L2L3 and L4L5 in a monotonic fashion.

Disc pathology at L3L4 level shows a positive monotonic correlation with LL only and shows a negative correlation with SS, PT, and PI [Figure 19].

Disc pathology at L5S1 level shows positive monotonic correlation with PT and LL [Figure 20]. Disc pathology at L5S1 however showed negative monotonic correlation with PI. This implies that with an increase in PI, disc pathology at L5S1 will always decrease; never remaining constant or increase.

A similar result was reported by Fei et al., ${ }^{[19]}$ Khallaf, ${ }^{[20]}$ and Oh and Eun et al. ${ }^{[21]}$

In our study, we took radiographs in a neutral standing position with knees straight and arms placed over the chest in such a way as to remove arms from the field of view. Imaging was acquired by using Digital Scannogram in LATERAL view from dorso-lumbar junction to the 


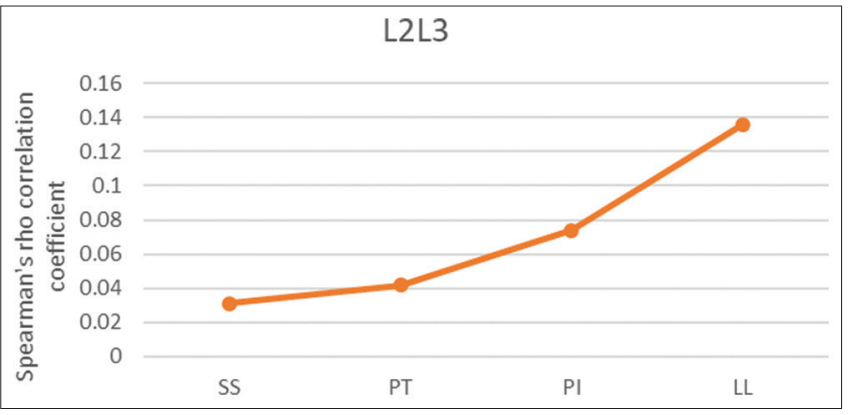

Figure 17: Correlation between disc pathology at L2L3 and spino-pelvic parameters

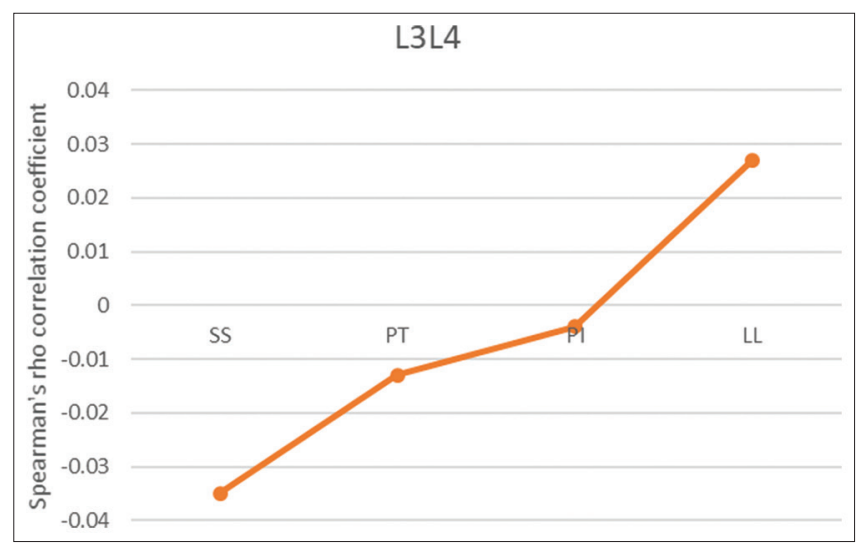

Figure 19: Correlation between disc pathology at L3L4 and spino-pelvic parameters

mid-thigh. Various spinopelvic parameters were measured from the scannogram by using computer software which required lines to be drawn manually on the DICOM file, but angles were measured automatically by software between lines drawn (Digimizer Image Analysis Software, Version 5.4.4; (C) 2005-2020 MedCalc Software Ltd). We measured SS, PT, PI, and LL independently. Mean values were - SS - $37.78^{\circ}, \mathrm{PT}-13.52^{\circ}$, and PI - 51.33 . Now, as we know PI must be equal to the sum of SS and PT i.e., $\mathrm{PI}=\mathrm{SS}+\mathrm{PT}$. So, $37.78+13.52(\mathrm{SS}+\mathrm{PT})$ gives 51.3 which is equal to mean PI we calculated 51.33. This proves that our radiography technique was fairly accurate. The technique of taking radiographs gave clear images which allowed us to clearly mark lines on computer software to calculate angles. This can be interpreted that our technique can be used as a standard technique to calculate spinopelvic parameters and whole spine radiographs are not a must-have to calculate these parameters (SS, PI, and PT). A similar observation was made in a study by Chung et al. ${ }^{[22]}$ who concluded that spinopelvic and LL measurements on lateral lumbar radiographs were similar to those on lateral whole-spine radiographs and exhibited excellent reproducibility.

\section{Conclusion}

Disc herniation has multifactorial pathophysiology. Correlation of sacropelvic parameters with disc herniation

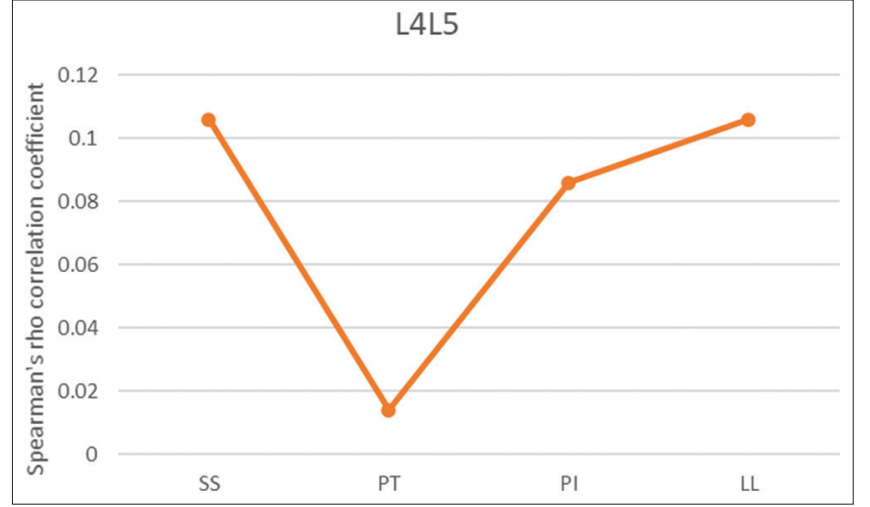

Figure 18: Correlation between disc pathology at L4L5 and spino-pelvic parameters

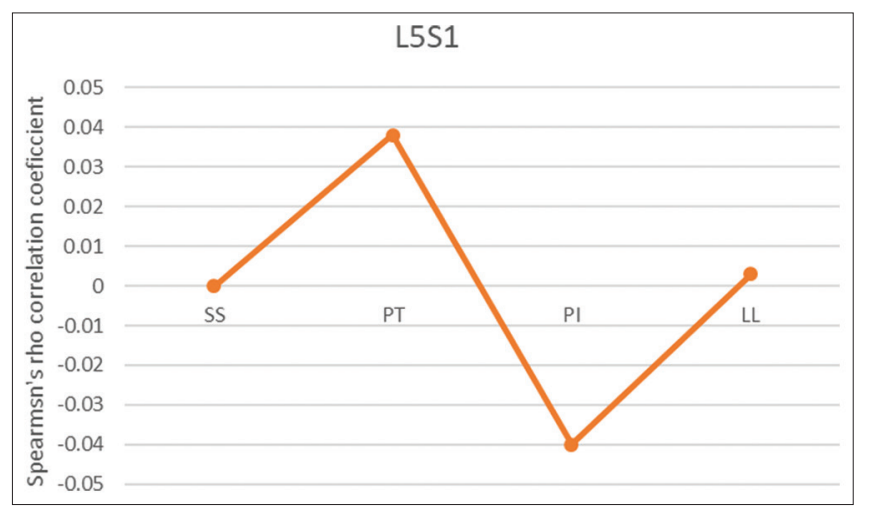

Figure 20: Correlation between disc pathology at L5S1 and spino-pelvic parameters

in the young population is a new paradigm for research and should be explored further with prospective randomized controlled studies to validate results. Standing lateral view radiograph from dorso-lumbar junction to the mid-thigh is as good as standing whole spine radiograph for measurement of spinopelvic parameters.

\section{Declaration of patient consent}

The authors certify that they have obtained all appropriate patient consent forms. In the form, the patient(s) has/have given his/her/their consent for his/her/their images and other clinical information to be reported in the journal. The patients understand that their names and initials will not be published and due efforts will be made to conceal their identity, but anonymity cannot be guaranteed.

\section{Financial support and sponsorship}

Nil.

\section{Conflicts of interest}

There are no conflicts of interest.

\section{References}

1. Jang JS, Lee SH, Min JH, Maeng DH. Influence of lumbar lordosis restoration on thoracic curve and sagittal position in lumbar 
degenerative kyphosis patients. Spine (Phila Pa 1976) 2009;34:280-4.

2. Kalichman L, Li L, Hunter DJ, Been E. Association between computed tomography-evaluated lumbar lordosis and features of spinal degeneration, evaluated in supine position. Spine J 2011;11:308-15.

3. Keorochana G, Taghavi CE, Lee KB, Yoo JH, Liao JC, Fei Z, et al. Effect of sagittal alignment on kinematic changes and degree of disc degeneration in the lumbar spine: An analysis using positional MRI. Spine (Phila Pa 1976) 2011;36:893-8.

4. Duval-Beaupère G, Schmidt C, Cosson P. A barycentremetric study of the sagittal shape of spine and pelvis: The conditions required for an economic standing position. Ann Biomed Eng 1992;20:451-62.

5. Singh R, Yadav SK, Sood S, Yadav RK, Rohilla R. Spino-pelvic radiological parameters in normal Indian population. Sicot J 2018;4:14.

6. Mac-Thiong J-M, Labelle H, Berthonnaud É, Betz RR, Roussouly P. Sagittal spinopelvic balance in normal children and adolescents. Eur Spine J 2007;16:227-34.

7. Berthonnaud É, Dimnet J, Roussouly P, Labelle H. Analysis of the sagittal balance of the spine and pelvis using shape and orientation parameters. J Spinal Disord Tech 2005;18:40-7.

8. Mac-Thiong J-M, Wang Z, de Guise JA, Labelle H. Postural model of sagittal spino-pelvic alignment and its relevance for lumbosacral developmental spondylolisthesis. Spine 2008;33:2316-25.

9. Barrey C, Jund J, Noseda O, Roussouly P. Sagittal balance of the pelvis-spine complex and lumbar degenerative diseases. A comparative study about 85 cases. Eur Spine J 2007;16:1459-67.

10. Stagnara P, DeMauroy JC, Dran G, Gonon G, Costanzo G, Dimnet J, et al. Reciprocal angulation of vertebral bodies in a sagittal plane: Approach to references for the evaluation of kyphosis and lordosis. Spine (Phila Pa 1976) 1982;7:335-42.

11. Duval-Beaupère G, Legaye J. Composante sagittale de la statique rachidienne. Rev Rhum 2004;71:105-19.
12. Boulay C, Tardieu C, Hecquet J, Benaim C, Mouilleseaux B, Marty C, et al. Sagittal alignment of spine and pelvis regulated by pelvic incidence: Standard values and prediction of lordosis. Eur Spine J 2006;15:415-22.

13. Roussouly P, Berthonnaud E, Dimnet J. Geometrical and mechanical analysis of lumbar lordosis in an asymptomatic population: Proposed classification. Rev Chir Orthop Reparatrice Appar Mot 2003;89:632-9 (in French).

14. Endo K, Suzuki H, Tanaka H, Kang Y, Yamamoto K. Sagittal spinal alignment in patients with lumbar disc herniation. Eur Spine J 2010;19:435-8.

15. Rajnics P, Templier A, Skalli W, Lavaste F, Illes T. The importance of spinopelvic parameters in patients with lumbar disc lesions. International Orthopaedics 2002;26:104-8.

16. Wang T, Wang H, Liu H, Ma L, Liu F-Y, Ding W-Y. Sagittal spinopelvic parameters in 2-level lumbar degenerative spondylolisthesis: A retrospective study. Medicine 2016;95:e5417.

17. Ferrero E, Ould-Slimane M, Gille O, Guigui P. Sagittal spinopelvic alignment in 654 degenerative spondylolisthesis. Eur Spine J 2015;24:1219-27.

18. Lai Q, Gao T, Lv X, Liu X, Wan Z, Dai M, et al. Correlation between the sagittal spinopelvic alignment and degenerative lumbar spondylolisthesis: A retrospective study. BMC Musculoskelet Disord 2018;19:151.

19. Fei H, Li WS, Sun ZR, Ma QW, Chen ZQ. Analysis of spino-pelvic sagittal alignment in Young Chinese patients with lumbar disc herniation. Orthop Surg 2017;9:271-6.

20. Khallaf ME. Three-dimensional analysis of spino-pelvic alignment in individuals with acutely herniated lumbar intervertebral disc. BMR 2017;30:759-65.

21. Oh Y-M, Eun J-P. Clinical impact of sagittal spinopelvic parameters on disc degeneration in young adults. Medicine 2015;94:e1833.

22. Chung N-S, Jeon C-H, Lee H-D, Won S-H. Measurement of spinopelvic parameters on standing lateral lumbar radiographs: Validity and reliability. Clin Spine Surg 2017;30:E119-23. 